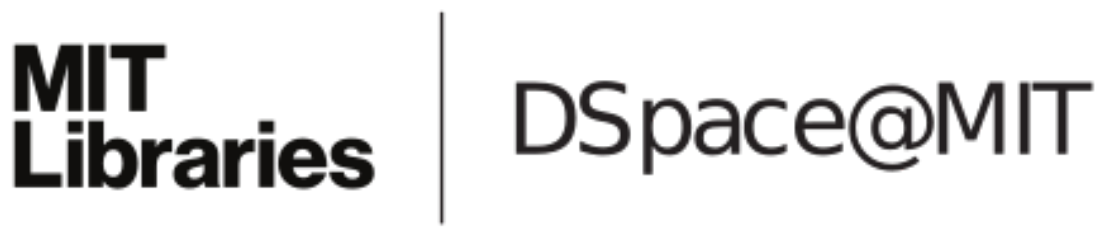

\author{
MIT Open Access Articles
}

Rectangular Symmetry Morphologies in a Topographically Templated Block Copolymer

The MIT Faculty has made this article openly available. Please share how this access benefits you. Your story matters.

Citation: Tavakkoli K. G., Amir, Adam F. Hannon, Kevin W. Gotrik, Alfredo AlexanderKatz, Caroline A. Ross, and Karl K. Berggren. "Rectangular Symmetry Morphologies in a Topographically Templated Block Copolymer." Advanced Materials 24, no. 31 (June 21, 2012): 4249-4254.

As Published: http://dx.doi.org/10.1002/adma.201104895

Publisher: Wiley Blackwell

Persistent URL: http://hdl.handle.net/1721.1/92712

Version: Author's final manuscript: final author's manuscript post peer review, without publisher's formatting or copy editing

Terms of use: Creative Commons Attribution-Noncommercial-Share Alike 


\section{Rectangular Symmetry Morphologies in a Topographically Templated Block Copolymer}

By Amir Tavakkoli K. G., Adam F. Hannon, Kevin W. Gotrik, Alfredo Alexander-Katz, Caroline A. Ross*, and Karl K. Berggren*

A. Tavakkoli K. G., Prof. K. K. Berggren

Department of Electrical Engineering and Computer Science,

Massachusetts Institute of Technology,

Cambridge, MA 02139, USA

E-mail: berggren@ mit.edu

A. Tavakkoli K. G.

NUS Graduate School for Integrative Sciences \& Engineering (NGS),

Singapore 117456

Prof. K. K. Berggren

Kavli Institute of Nanoscience, Technical University of Delft, Delft, NL

A. F. Hannon, K. W. Gotrik, Prof. A. Alexander-Katz, Prof. C. A. Ross

Department of Materials Science and Engineering,

Massachusetts Institute of Technology,

Cambridge, MA 02139, USA

E-mail: caross@mit.edu

Keywords: Block copolymer; Hybrid patterns, Templated self-assembly, Graphoepitaxy, Square pattern

Templated self-assembly of block copolymer (BCP) thin films can enhance the resolution and throughput of nanoscale lithography processes. ${ }^{[1-18]}$ This method has been used to produce large-area defect-free lamellar, cylindrical, or spherical microdomain patterns through chemical $^{[1-6]}$ or topographical ${ }^{[7-15]}$ templating. However, the formation of complex patterns with multiple morphologies in one BCP film (e.g. coexisting cylinders and spheres) requires additional process steps such as sequential cross-linking and solvent anneals. ${ }^{[19,20]}$ The period of the patterns is determined by the BCP chain length, and sub-10-nm-period (sub-5-nm halfpitch) patterns have been reported from low molecular weight BCPs. ${ }^{[8]}$ While a hexagonal lattice of microdomains is readily obtained from a diblock copolymer, obtaining a square symmetry pattern requires 1:1 templating of a diblock copolymer ${ }^{[21]}$, or use of a triblock terpolymer $^{[22]}$ or a blend of diblock copolymers ${ }^{[23]}$. We show that by using an array of majority-block-functionalized posts, it is possible to locally control the morphology of a 
diblock copolymer, achieve several morphologies simultaneously on a single substrate, and create patterns with square or rectangular symmetry. As a side benefit of this method, because the topographic template features are incorporated into the final pattern, the resulting spacing between the template features and the minority microdomains is smaller than the BCP period, i.e. the areal density of features is increased. Three-dimensional self-consistent-field-theory (SCFT) simulations were performed to calculate equilibrium morphologies and to explore a wider parameter space than that of the experiments.

Figure 1 shows a schematic diagram of the major steps of the experimental method. In the first step, the templates were fabricated by means of electron-beam-lithography (EBL) exposure of hydrogen silsesquioxane (HSQ) resist, then chemically functionalized with the majority-block brush, hydroxyl-terminated-polystyrene by grafting the hydroxyl-terminated ends onto the silica substrate. Next, poly(styrene-b-dimethylsiloxane) (PS-b-PDMS, $45.5 \mathrm{~kg}$ $\mathrm{mol}^{-1}$ ) was spin-coated onto the template substrates. The BCP used in this experiment had a bulk morphology consisting of PDMS cylinders in a PS matrix with an equilibrium periodicity of $\sim 35 \mathrm{~nm}$. Annealing of the BCP thin film was done at room temperature using solvent vapor. $\mathrm{A} \mathrm{CF}_{4}$ reactive-ion etch (RIE) was done to remove the top PDMS surface layer and immediately followed by an oxygen RIE to remove the PS matrix and leave the oxidizedPDMS (ox-PDMS) patterns on the substrates.

Figure 2 shows a scanning electron micrograph (SEM) of a hybrid BCP pattern on a substrate. The square array of PS-functionalized posts had 27-nm diameter and 73-nm period in both the $x$ - and $y$-directions. Two different BCP morphologies, cylinders outside the template region and a square symmetry perforated lamella within it, appeared on the substrate.

By using different template conditions, other morphologies could be achieved. Figure 3a-h shows SEMs of the morphologies of the BCP for different post periods. By varying the post period from less than $L_{0}$ (the equilibrium BCP period) to more than $2 L_{0}$, different morphologies were observed, such as cylinders (Figure 3a,h), undulated cylinders (Figure 3b), 
spheres (Figure 3c), ellipsoids (Figure 3d), periodic superstructures (Figure 3e), perforated lamellae with a perforation at each post (denoted by L1) (Figure 3f) and perforated lamellae with additional perforations between the posts (denoted by L2) (Figure 3g). Most of these morphologies were observed at a particular post period in the $x$ - and $y$-directions; as the period varied around these values, the uniformity of the structures decreased. The periods where each of these morphologies occurred depended on the post diameters. Figure $3 \mathrm{i}$ shows the experimental phase diagram plotted against post period $P_{\mathrm{x}}$ and $P_{\mathrm{y}}$ varying from $0.5 L_{0}$ to $2.5 L_{0}$ for a post diameter of $10 \mathrm{~nm}$ to $20 \mathrm{~nm}$. We label $P_{\mathrm{y}}$ as the smaller period in rectangular lattices. In this figure, each data point represents a sample in which one morphology covering more than $70 \%$ of the templated area. A black triangle represents a sample with no dominant morphology.

SCFT was used to model the morphology resulting from post periods of $0.7 L_{0}$ to $2.0 L_{0}$. A phase diagram of the simulation results is shown in Figure $3 \mathrm{j}$. The general trends of the simulations showed the same morphologies observed in experiments, including cylinders (yellow region in Figure 3j), spheres (blue region in Figure 3j), ellipsoids (green region in Figure 3j), perforated lamellae L1 (pink region in Figure 3j), and L2 (orange region in Figure 3j), all near the same post period regimes seen experimentally. The simulations can yield both equilibrium and metastable structures, so if the model results differed from those observed experimentally, multiple simulations were performed to determine the energy minimum. The phase diagram shows the state with lowest energy. Additional larger unit cell simulations were done with a post period of $P_{\mathrm{x}}=P_{\mathrm{y}}=2.29 L_{0}$ for comparison with Figure $3 \mathrm{~h}$. The simulations showed that both L2 and double cylinders had similar energies for these post periods (inset image in Figure 3j).

The simulations assume periodic boundary conditions, whereas the real systems have a finite post lattice area and the equilibrium cylinder structures surrounding the post lattice boundaries might influence the morphologies in the templated region. Also, the simulation 
does not address local film thickness changes caused by solvent annealing, and since the simulations only yield saddle point solutions and do not reproduce realistic kinetics, these structures will not correspond exactly to the experiment in cases where kinetically trapped structures form. Despite this, the simulations give a very good qualitative agreement with the experiment, and could therefore be predictively useful. Two major discrepancies occurred between the model and experiment. First, in cases where experimentally the cylinders oriented parallel to the axis with larger period with $P_{\mathrm{x}}>L_{0}$ and $P_{\mathrm{y}}<L_{0}$, the model predicted that this was a higher energy state than alignment parallel to the axis with smaller period. Second, the model could not reproduce the superstructures (Fig. 3e) because it included only one lattice period.

We now describe the various morphologies in more detail. When $P_{\mathrm{x}}$ was similar to or greater than $L_{0}$ and $P_{\mathrm{y}}<L_{0}$, the PDMS cylinders oriented along the $y$-direction, Figure $3 \mathrm{a}$. The cylinders in the templated region showed a small amount of necking or undulation near the posts, and a different width compared to those in the untemplated region: when $P_{\mathrm{x}}>L_{0}$ the width of the cylinders was larger than the untemplated case. When $P_{\mathrm{x}}$ was similar to or greater than $L_{0}$ and $P_{\mathrm{y}}=\sim L_{0}$, the PDMS cylinders oriented along the $x$-direction, Figure $3 \mathrm{~b}$. The cylinders showed distinct undulations caused by the PS-coated posts. These cylinder morphologies occurred over a wide range of $P_{\mathrm{x}}$ in both experiment and model. The SCFT examples in Fig. 3j show only small fluctuations in the cylinder width, but cylinders with larger undulations oriented along the commensurate direction appeared for shorter or thinner posts or a thicker BCP film.

Figure $3 \mathrm{c}$ shows a spherical morphology with a square symmetry that occurred when $P_{\mathrm{x}}=P_{\mathrm{y}}$ and the post period was incommensurate with $L_{0}$. Figure $\mathrm{S} 1$ in the Supporting Information shows the change from a cylindrical morphology to a square lattice spherical morphology by increasing the post period. By increasing the post period in both directions, a condition was reached in which the $\mathrm{BCP}$ was commensurate with the lattice under a rotation 
of 45 degrees, which resulted in an elliptical morphology for the PDMS, as seen in Figure 3d. Figure $3 \mathrm{i}$ shows that the simulated spherical and ellipsoidal morphologies occurred within a small range of post periods, and the ellipsoidal structures were oblate ellipsoids rather than the 45-degree-oriented prolate ellipsoids observed in the experiment. The higher symmetry in the simulation may result from the periodic boundary conditions which do not provide a preferential in-plane direction for the ellipsoids to orient.

Figure $3 \mathrm{e}$ shows a periodic superstructure of cylinders and ellipsoids. Periodic superstructures of cylinders and ellipsoids or cylinders and spheres were observed within a large region of post periodicity, in between template periods that generated either cylinders or perforated lamellae, as shown in Figure 3i. These superstructures appear to be metastable combinations of the ellipsoidal and cylindrical morphologies. Such periodic patterns were only produced in SCFT simulations of double cells in which one of the cells was seeded with initial conditions consisting of a sphere of PDMS. The simulations suggest that spheres or ellipsoids have similar energies to cylinders (cylinders have a slightly higher energy) in this regime of post period, and this indicates that the superstructure represents a combination of these states. Formation of a cylinder may promote formation of ellipsoids in the adjacent unit cells, which then favors formation of another cylinder, etc., building up the superstructure. Energy differences between the superstructure and cylinder-only morphologies are small, for example the superstructure had $0.034 \%$ higher energy for $P_{\mathrm{x}}=1.0 L_{0}$ and $P_{y} \sim 1.5 L_{0}$ in the simulations.

Figure 3f shows a perforated lamellar morphology L1 which occurred when the post periods in the $x$-, $y$-, and diagonal directions were between $L_{0}$ and $2 L_{0}$. The volume of the oxPDMS between the posts was intermediate between that of one cylinder and two cylinders. Figure S2 in the Supporting Information shows the appearance of perforated lamellae from superstructures by increasing the post period. 
Figure $3 \mathrm{~g}$ shows perforated lamellae with additional perforations between the posts (L2) which formed for $P_{\mathrm{x}}=P_{\mathrm{y}}=1.8-2.3 L_{0}$; for larger period, the structure transitioned to pairs of cylinders lying between the posts, Figure 3-h. The SCFT simulations showed perforated lamellae L2 for $P_{\mathrm{x}}=P_{\mathrm{y}}>1.7 L_{0}$, but the model did not reproduce the gradual transition between morphologies. Larger multi-cell simulations may show such transitional structures. Larger unit cells (e.g. $\left.P_{\mathrm{x}}=P_{\mathrm{y}}=2.29 L_{0}\right)$ generated either double parallel cylinders or perforated lamellae L2, Figure 3j (inset), and these two structures had degenerate energies.

Considering that both the HSQ template features and the ox-PDMS are silica-based structures with high etch resistance, they both constitute the final pattern produced by the templated BCP. The areal density of HSQ plus ox-PDMS features in the final pattern was therefore higher compared to the density of ox-PDMS features in an untemplated film. For example, the feature spacing along the $\mathrm{x}$-axis in Figure 2a, or the $\mathrm{y}$-axis in Figure 2b, is $17 \mathrm{~nm}$, half of $L_{0}$. This process therefore achieves not only control over the film morphology and formation of square and rectangular symmetry patterns, but a denser set of etch-resistant features than is possible from the BCP alone.

The morphological control was further illustrated by templating a PS-PDMS BCP with low molecular weight $\left(16 \mathrm{~kg} \mathrm{~mol}^{-1}\right)$. This material formed in-plane cylinders with spacing $17 \mathrm{~nm}$ on a smooth substrate, but a square array of posts with $25 \mathrm{~nm}$ period templated a square symmetry pattern of ox-PDMS spheres between the posts, Figure 4. The ox-PDMS spheres and the posts together formed a square pattern with feature spacing of $25 / \sqrt{2}=17 \mathrm{~nm}$, similar to Figure $2 \mathrm{c}$ but smaller in dimensions.

We next show how the size of the ox-PDMS microdomains can be predicted analytically based on the geometry of the template and volume fraction of the BCP [for further details of the calculations, see Supporting Information]. Consider first a film, which forms a single layer of cylindrical PDMS microdomains with radius $R_{0}$ on a smooth substrate. 
When templated into spherical microdomains of radius $R_{\mathrm{S}}$ by an array of posts (as in Figures $2 \mathrm{c}$ or 4$)$,

$R_{s}=\left(\frac{3 R_{0}^{2}}{4 L_{0}}\left[P_{x} P_{y} \quad(r+T)^{2}\right]\right)^{\frac{1}{3}}$

where $r$ is the radius of the posts, and $T$ the thickness of the PS brush layer on the posts.

For the case of a bilayer BCP film which forms two layers of cylinders on a smooth substrate, if $R_{01}$ and $R_{02}$ are the radii of the ox-PDMS cylinders in the top and bottom layers of microdomains, then in cases where post templating forms a monolayer of spheres of radius $R_{\mathrm{s}}$, $R_{s}=\left(\frac{3\left(R_{01}^{2}+R_{02}^{2}\right)}{4 L_{0}}\left[P_{x} P_{y} \quad(r+T)^{2}\right]\right)^{\frac{1}{3}}$.

These results show that the radius $R_{\mathrm{S}}$ of the templated spherical PDMS microdomains depends on both the volume fraction of PDMS (which determines $R_{0}$ for a given $L_{0}$ ) and the geometry of the template. Figure 5a-c shows the experimental verification of this analysis for three different conditions, in which different post geometries, film thicknesses and molecular weights $(45 \mathrm{~kg} / \mathrm{mol}$ and $16 \mathrm{~kg} / \mathrm{mol})$ are used. Figure 5a shows a film consisting of a monolayer of cylinders on a smooth substrate, and Figures $5 \mathrm{~b}$ and $5 \mathrm{c}$ are from bilayers of cylinders. In Figures 5a-c, the square post lattice yielded PDMS spheres whose predicted diameters from Eq. 1 and 2 were $24 \mathrm{~nm}, 11 \mathrm{~nm}$ and $33 \mathrm{~nm}$ respectively, which compares well with the measured values of $24 \mathrm{~nm}, 11 \mathrm{~nm}$, and $32 \mathrm{~nm}$. The agreement supports the interpretation of the templated morphologies as consisting of a monolayer of PDMS spheres, consistent with the SCFT model. Figure 5d shows a non-square post lattice in which resulted in ellipsoids rather than spheres.

The central result of this article is the use of an array of majority-block-functionalized topographical posts to control the self-assembly of a $\mathrm{BCP}$, allowing a variety of morphologies to be produced, including arrays of spheres, ellipsoids, cylinders, undulating cylinders, perforated lamellae and superstructures, by varying the geometrical parameters of the post 
array. This is useful for generation of complex patterns, which is an objective for nano-device fabrication. The total density of the ox-PDMS and HSQ features in the etched structure is higher than the density of ox-PDMS features in an untemplated film, and the process therefore increases areal density by placing HSQ features within the PS majority domains. Threedimensional SCFT simulations of morphology vs. template geometry gave a good agreement with the observed cases for most post periods.

\section{Experimental Section}

Template fabrication: The templates were fabricated by EBL of HSQ (Dow Corning XR-1541 $2 \%$ solids), a negative-tone electron resist. HSQ was spin-coated on Si (100) wafers at different thicknesses $(21 \mathrm{~nm}, 27 \mathrm{~nm}$, and $38 \mathrm{~nm})$ depending on the BCP being used. The thickness of the spin-coated HSQ was measured using an ellipsometer. A Raith 150 EBL tool at $30 \mathrm{kV}$ acceleration voltage and $300 \mathrm{pA}$ beam current was used for the exposure of the resist. Arrays of HSQ posts with diameters ranging from $10 \mathrm{~nm}$ to $30 \mathrm{~nm}$ (measured by image processing software) were obtained by using different doses ranging from $10 \mathrm{fC}$ to $70 \mathrm{fC}$. After exposure, samples were developed in a salty developer [24] for 4 minutes followed by a rinse in de-ionized water for 2 minutes. Subsequently, oxygen plasma asher (50w, 0.35 Torr) was used to remove possible organic residues and to change the HSQ patterns to silica. Block copolymer self assembly: The templates were chemically functionalized with a hydroxyl-terminated PS brush $\left(3 \mathrm{~kg} \mathrm{~mol}^{-1}\right.$ and $1 \mathrm{~kg} \mathrm{~mol}^{-1}$ for high and low molecular weight of BCPs, respectively) by first spinning the PS solution onto the templates and then placing them in a vacuum oven ( 20 torr) at $170^{\circ} \mathrm{C}$ for 16 hours and subsequently rinsing with toluene to remove ungrafted polymer chains. Then poly(styrene-b-dimethylsiloxane) (PS-b-PDMS) (high MW: $45.5 \mathrm{~kg} \mathrm{~mol}^{-1}, f_{\mathrm{PDMS}}=32 \%$ or low $\mathrm{MW}: 16 \mathrm{~kg} \mathrm{~mol}^{-1}, f_{\mathrm{PDMS}}=31 \%$ ) was spun onto the HSQ templates to a thickness of $29 \mathrm{~nm}$ or $25 \mathrm{~nm}$, respectively. Annealing of the high MW BCP thin film was done by placing the samples in a chamber in the presence of a cosolvent 
vapor resulting from a mixture of heptane and toluene liquids $\left(V_{\mathrm{To}} / V_{\mathrm{Hep}}=5\right)$ which swelled both blocks of the copolymer and improved the kinetics of self-assembly [25]. After $3 \mathrm{hr}$ anneal followed by quenching in air and etching, the film period was around $35 \mathrm{~nm}$. For the low MW BCP, a saturated vapor of acetone was used in a closed dish for $5 \mathrm{hr}$ resulting in a BCP period of around $17 \mathrm{~nm}$. A $5 \mathrm{~s}$ (high MW) or $2 \mathrm{~s}$ (low MW) $\mathrm{CF}_{4} \mathrm{RIE}$ (50 W, 15 mTorr) was done to remove the top PDMS surface wetting layer, immediately followed by a $22 \mathrm{~s}$ (high MW) and $12 \mathrm{~s}$ (low MW) oxygen RIE (6 mTorr, $90 \mathrm{~W}$ ) to remove the PS matrix and leave the ox-PDMS patterns. Scanning electron micrographs (SEMs) were obtained using a Raith 150 scanning electron microscope operated with an acceleration voltage of $10 \mathrm{kV}$. Simulation methods:Using the same methods as presented by Mickiewicz et.al [26], threedimensional SCFT simulations were done to compare the equilibrium morphologies found in the experiment with those in the simulations. The simulations were used to explore a wider parameter space than allowed in the experiments in order to predict the commensurability conditions for the various morphologies. In the simulations, the system was modeled using three dimensional unit cells with periodic boundary conditions in the $x$ - and $y$-directions as schematically depicted in Figure S4. The posts were modeled by constraining the fields in the region of the posts to a large value that prevents polymer density from evolving in that region. The brush layer was modeled using a field constraint around the boundary of the post region that was attractive to the majority block (PS) and repulsive to the minority block (PDMS). The top air interface was modeled similar to the brush layer but preferentially attractive to the PDMS as experimental observation shows PDMS to have a lower surface energy with air than PS. To match experimental parameters, the model used a value of $\chi N=$ 30.0 and $f_{\mathrm{PDMS}}=0.32$ where $N$ in the simulation is the number of coarse-grained statistical monomers. The system was evolved starting with random initial field conditions until a saddle point solution to the field equations was obtained that was either an equilibrium or metastable morphology. 


\section{Acknowledgements}

The authors acknowledge support from the Semiconductor Research Corporation, the FENA Center, NRI, SMA, National Science Foundation, Taiwan Semiconductor Manufacturing Company and Tokyo Electron. A.T.K.G would like to express his sincere gratitude to Professor T. C. Chong and Dr. S. N. Piramanayagam for their support, and would like to acknowledge his fellowship from NGS Singapore. The Research Laboratory of Electronics Scanning-Electron-Beam Lithography Facility provided facilities for this work. The authors also thank M. Mondol and J. Daley for technical assistance.

Supporting Information is available online from Wiley InterScience or from the author.

[1] R. Ruiz, H. Kang, F. A. Detcheverry, E. Dobisz, D. S. Kercher, T. R. Albrecht, J. J. de Pablo, P. F. Nealey, Science 2008, 321, 936.

[2] J. Y. Cheng, C. T. Rettner, D. P. Sanders, H. Kim, W. D. Hinsberg, Advanced Materials 2008, 20, 3155 .

[3] S. O. Kim, H. H. Solak, M. P. Stoykovich, N. J. Ferrier, J. J. De Pablo, P. F. Nealey, Nature 2003, 424, 411.

[4] M. P. Stoykovich, M. Müller, S. O. Kim, H. H. Solak, E. W. Edwards, J. J. De Pablo, P. F. Nealey, Science 2005, 308, 1442.

[5] L. Rockford, Y. Liu, P. Mansky, T. P. Russell, Phys. Rev. Lett. 1999, 82, 2602.

[6] S. G. Xiao, X. M. Yang, S. Park, D. Weller, T. P. Russell, Adv. Mater. 2009, 21, 2516.

[7] J. K. W. Yang, Y. S. Jung, J. B. Chang, R. A. Mickiewicz, A. Alexander-Katz, C. A. Ross, K. K. Berggren, Nature Nanotechnology 2010, 5, 256.

[8] S. Park, D. H. Lee, J. Xu, B. Kim, S. W. Hong, U. Jeong, T. Xu, T. P. Russell, Science 2009, 323, 1030. 
[9] J. G. Son, A. F. Hannon, K. W. Gotrik, A. Alexander-Katz, C. A. Ross, Adv. Mater. 2011, 23,634 .

[10] R. A. Segalman, H. Yokoyama, E. J. Kramer, Adv. Mater. 2001, 13, 1152.

[11] J. Y. Cheng, C. Ross, E. L. Thomas, H. I. Smith, Vancso, G. J. Adv. Mater. 2003, 15, 1599.

[12] C. T. Black, O. Bezencenet, Nanotechnology, IEEE Transactions on 2004, 3, 412.

[13] R. Ruiz, N. Ruiz, Y. Zhang, R. L. Sandstrom, C. T. Black, Adv. Mater. 2007, 19, 2157.

[14] D. Sundrani, S. B. Darling, S. J. Sibener, Nano Lett. 2004, 4, 273.

[15] R. Ruiz, R. L. Sandstrom, C. T. Black, Adv. Mater. 2007, 19, 587.

[16] S. Xiao, X. M. Yang, K. Y. Lee, R. J. ver der Veerdonk, D. Kuo, T. P. Russell, Nanotechnology 2011, 22, 305302.

[17] S.-J. Jeong, H.-S. Moon, B. H. Kim, J. Y. Kim, J. Yu, S. Lee, M. J. Lee, H. Y. Choi, S. O. Kim, ACS Nano 2010, 4, 5181.

[18] Q.-Y. Tang, Y.-Q. Ma, Soft Matter, 2010, 6, 4460.

[19] J. K. Bosworth, C. T. Black, C. K. Ober, ACS Nano 2009, 3, 1761.

[20] J. G. Son, J.-B. Chang, K. K. Berggren, C. A. Ross, Nano lett. 2011, 11, 5079.

[21] S.-M. Park, G. S. W. Craig, Y.-H. La, H. H. Solak, P. F. Nealey, Macromolecules 2007, 40, 5084.

[22] J. G. Son, J. Gwyther, J. B. Chang, K. K. Berggren, I. Manners, C. A. Ross, Nano Lett. 2011, 11, 2849.

[23] C. Tang, E. M. Lennon, G. H. Fredrickson, E. J. Kramer, C. J. Hawker, Science 2008, 322, 429.

[24] J. K. W. Yang, K. K. Berggren, J. Vac. Sci. Tech. B 2007, 25, 2025.

[25] Y. S. Jung, C. A. Ross, Advanced Materials 2009, 21, 2540.

[26] R. A. Mickiewicz, J. K. W. Yang, A. F. Hannon, Y. S. Jung, A. Alexander-Katz; K. K. Berggren, C. A. Ross, Macromolecules 2010, 43, 8290. 


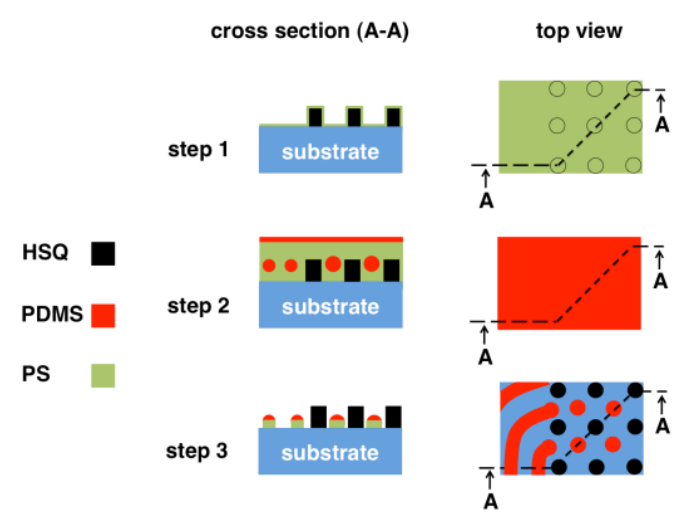

Figure 1. Schematic diagram showing the major steps of the fabrication process. Step 1: defining the post template by EBL and chemically functionalizing with a PS brush; step 2: spin-coating and annealing of the BCP; step 3: removing the PDMS top wetting layer by $\mathrm{CF}_{4} \mathrm{RIE}$ and then the PS matrix by oxygen RIE, then imaging in SEM. The height of the oxidized PDMS cylinders in step 3 is expected to be lower than that of the as-annealed PDMS cylinders in step 2 due to the etch process.

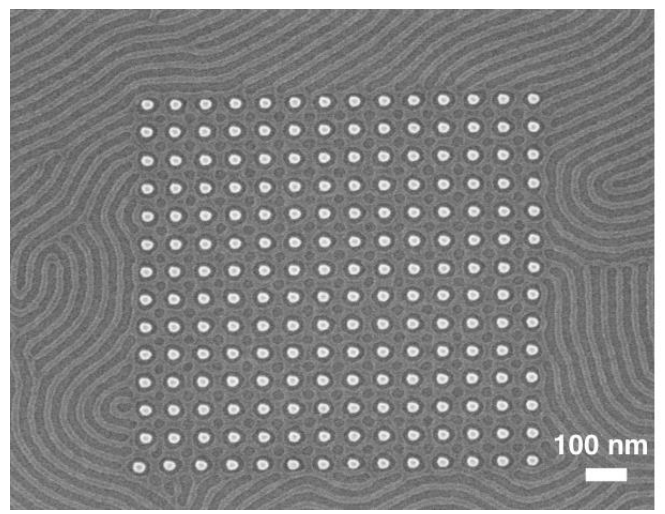

Figure 2. SEM of a hybrid BCP pattern on a substrate. White and light grey shades represent HSQ and ox-PDMS, respectively. Inside the templated region, the ox-PDMS formed a perforated lamella and outside of it, it formed cylinders. 


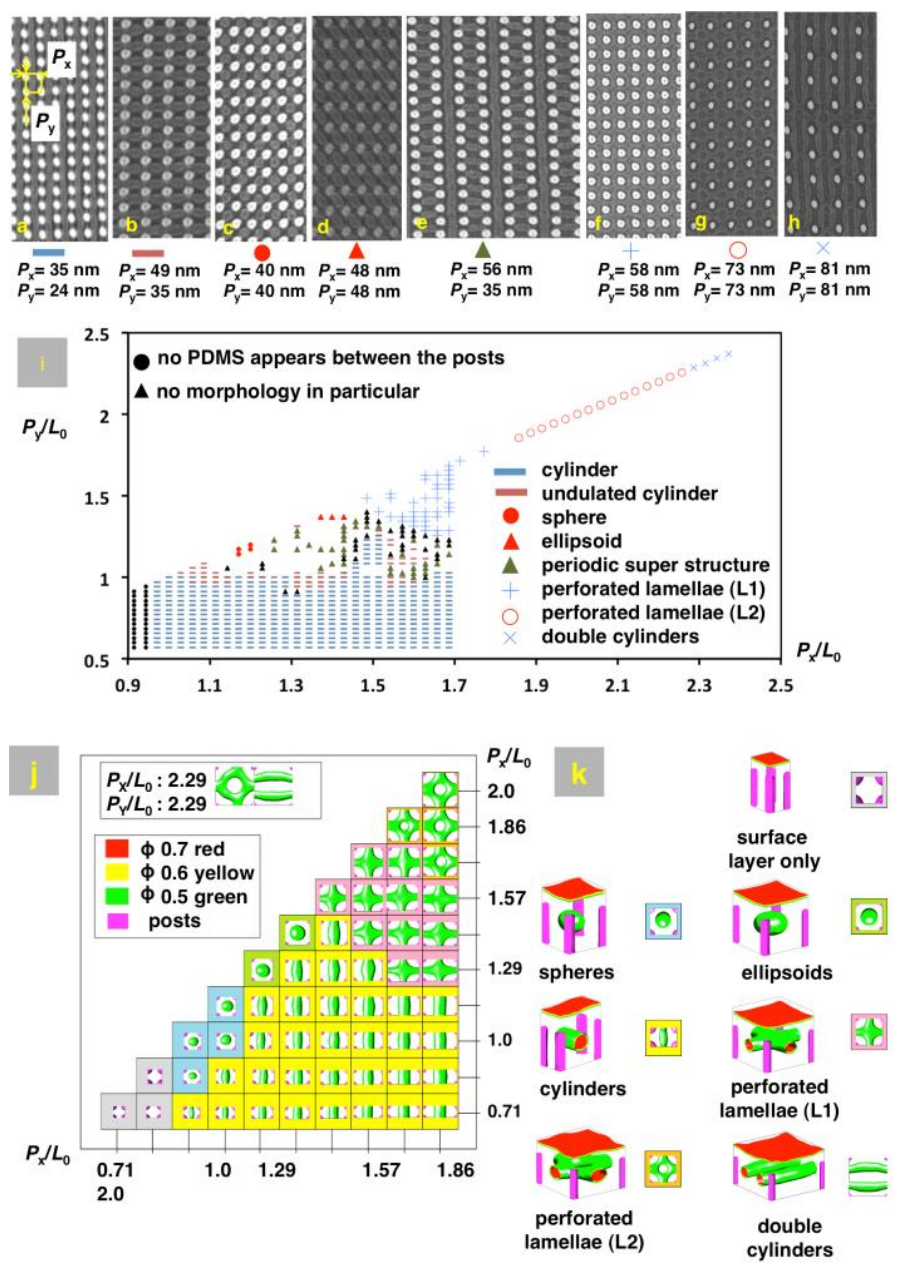

Figure 3. (a-h) SEMs of different morphologies of PDMS microdomains. The HSQ appears white and the ox-PDMS light grey. The observed morphologies are: (a) cylinders, (b) undulated cylinders, (c) spheres, (d) ellipsoids, e) periodic superstructures, (f) perforated lamellae L1, (g) perforated lamellae L2, (h) double cylinders. (i) A phase diagram showing a summary with each data point representing one sample. (j) Phase diagram using 3D SCFT modeling. The horizontal axis is the reduced post spacing distance in the $x$-direction $P_{x} / L_{0}$ and the vertical axis is the reduced post spacing distance in the $y$-direction $P_{y} / L_{0}$. The structures are shown from the top after removing the PDMS surface layer, with only the $\phi=0.5$ surfaces shown in green. (k) 3D SCFT simulation results showing contours of $\phi$ $=0.5$ (green), 0.6 (yellow) and 0.7 (red), where $\phi$ represents the normalized density of the PDMS.

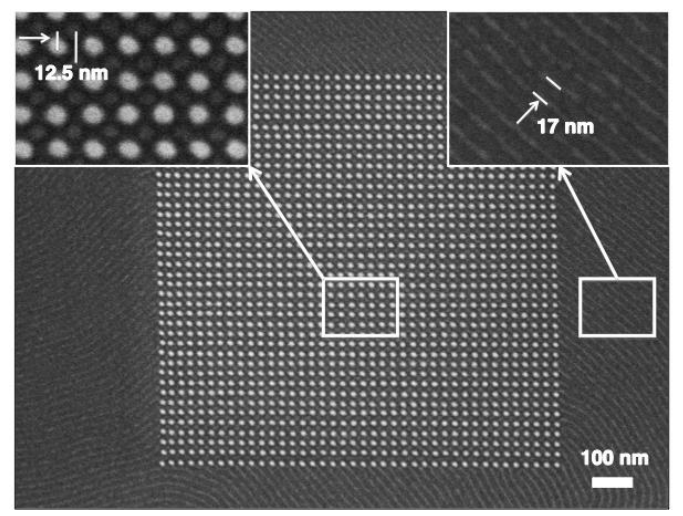

Figure 4. SEM of a high-resolution hybrid BCP pattern on a substrate. White and light grey colors represent HSQ and ox-PDMS, respectively. In the template region, ox-PDMS microdomains are spheres and outside of it, they are cylinders. (Insets: Zoomed-in images of areas inside and outside the template) 


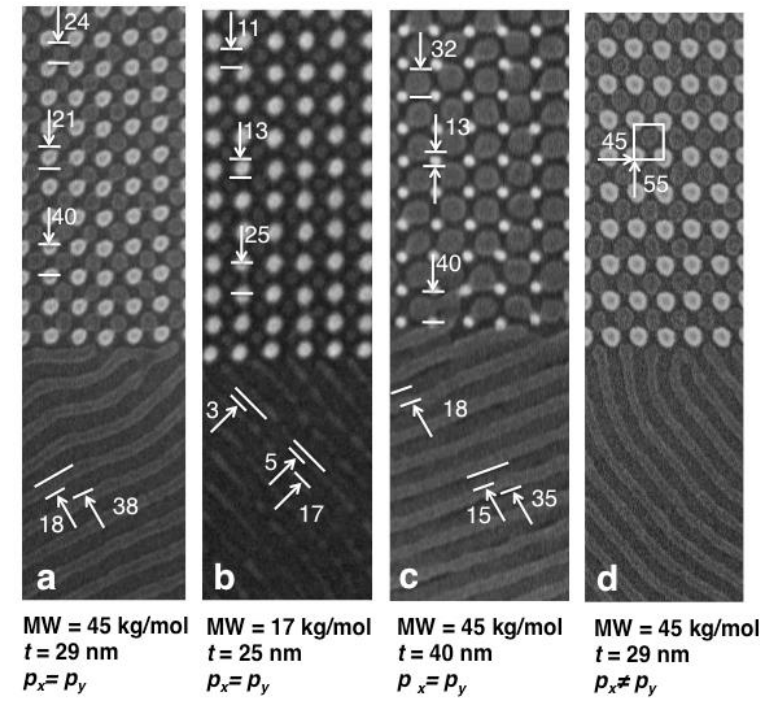

Figure 5. SEMs illustrating the change of the morphology of ox-PDMS cylinders to spheres using a PS-functionalized template. White and light grey colors indicate HSQ posts and ox-PDMS microdomains, respectively. The unit of numbers in all images is $\mathrm{nm}$ and for images (a-c), a square lattice template was used. These images are the results of (a) a single layer BCP with a molecular weight of $45 \mathrm{~kg} \mathrm{~mol}^{-1}$ (b) a bilayer BCP with a molecular weight of $16 \mathrm{~kg} \mathrm{~mol}^{-1}$ and (c) a bilayer BCP with a molecular weight of $45 \mathrm{~kg} \mathrm{~mol}^{-1} \mathrm{~d}$ ) a single layer BCP with a molecular weight of $45 \mathrm{~kg} \mathrm{~mol}^{-1}$ and a rectangular lattice template. 